\title{
Energy-resolved X-ray detectors: the future of diagnostic imaging
}

\author{
This article was published in the following Dove Press journal: \\ Reports in Medical Imaging \\ 19 January 2015 \\ Number of times this article has been viewed
}

\section{Danilo Pacella \\ ENEA-Frascati, Rome, Italy}

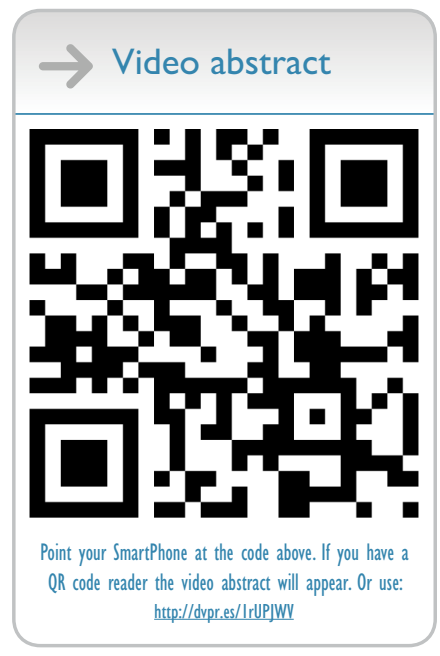

Correspondence: Danilo Pacella Via E Fermi 45, 00044 Frascati, Rome, Italy

Tel +390694005707

$\mathrm{Fax}+390694005135$

Email danilo.pacella@enea.it
Abstract: This paper presents recent progress in the field of X-ray detectors, which could play a role in medical imaging in the near future, with special attention to the new generation of complementary metal-oxide semiconductor (C-MOS) imagers, working in photon counting, that opened the way to the energy-resolved X-ray imaging. A brief description of the detectors used so far in medical imaging (photographic films, imaging plates, flat panel detectors), together with the most relevant imaging quality parameters, shows differences between, and advantages of these new C-MOS imagers. X-ray energy-resolved imaging is very attractive not only for the increase of contrast but even for the capability of detecting the nature and composition of the material or tissue to be investigated. Since the X-ray absorption coefficients of the different parts or organs of the patient (object) are strongly dependent on the X-ray photon energy, this multienergy ("colored") X-ray imaging could increase enormously the probing capabilities. While dual-energy imaging is now a reality in medical practice, multienergy is still in its early stage, but a promising research activity. Based on this new technique of color X-ray imaging, the entire scheme of source-object-detector could be revised in the future, optimizing spectrum and detector to the nature and composition of the target to be investigated. In this view, a transition to a set of monoenergetic X-ray lines, suitably chosen in energy and intensity, could be envisaged, instead of the present continuous spectra.

Keywords: X-ray detectors, X-ray medical imaging, C-MOS imagers, dual and multienergy CT

\section{Introduction}

The aim of this paper is to review recent progress in the field of X-ray detectors, which could play a role in medical imaging in the near future. In particular, this overview will focus on two subjects: first one is the new generation of complementary metal-oxide semiconductor (C-MOS) imagers, working in photon counting, that opened the way to the energy-resolved X-ray imaging; the second one is a better match between X-ray spectrum, object to be imaged, and detectors. These new techniques represent the leading edge of scientific research, and they have not yet well-established applications beyond the domain of the science. Despite that, these two topics represent the guidelines and the challenges for the evolution of X-ray medical imaging in the coming years.

Before entering into discussions of these items, a brief review of the detectors used so far in medical imaging will be done, with a cross-comparison of their features, showing advantages and limitations. In the next section, the physical parameters used to characterize any X-ray detector will be discussed. Then a description of analog detectors will be done, followed by a short review and comparisons of the digital ones, commercially available since a few years. The new generation of C-MOS imagers 
will be discussed in greater detail, seen in the perspective of their applications for medical imaging. Energy-discriminated imaging will be discussed in more detail, being one of the two core subjects of the paper. The second topic will be a review of the general scheme of the X-ray radiography in order to understand which directions of optimization and improvements in the diagnostics capabilities should be addressed. For a brief history of medical applications of X-ray, see the short note of Linton. ${ }^{1}$

In the current paper these subjects are considered from the point of view of the physical processes occurring in the production of X-ray, in their interactions with the matter (object to be investigated), and in their detection, not from a strictly clinical point of view. Nevertheless, the paper is addressed to professionals working in medical imaging domain, in the hope to provide an overview about the potential developments in this area. Physical processes and parameters are described to be easily understood by a nonspecialist reader.

\section{General features of X-ray imaging and detectors $X$-ray tubes}

$\mathrm{X}$-rays in medical applications are produced by cathodic tubes. Electrons, generated by a filament and then accelerated by an electric field (by means of an applied peak voltage $V_{\text {p }}$, whose value arrives up to $150 \mathrm{kV}_{\mathrm{p}}$ ), strike an anode (positive polarity). ${ }^{2}$ These high-energy electrons interact with the atoms of the anode through two dominant processes generating X-rays: braking radiation (bremsstrahlung) and atom inner excitation with following emission of fluorescence radiation. The first mechanism produces a continuous spectrum, while the second one emits a line radiation at fixed energy. In medical applications, for medium-high energy, a tungsten anode is used, and the outcoming spectrum is mainly continuous. For radiography at $70 \mathrm{kV}_{\mathrm{p}}$, a filter of $2.5 \mathrm{~mm}$ of aluminum is placed at the exit of the tube to cut the low-energy X-ray emissions (below 20-25 keV) off. For chest or trunk radiography, $120-150 \mathrm{kV}_{\mathrm{p}}$ is used, with $3 \mathrm{~mm}$ of $\mathrm{Al}$ (cutoff at $30 \mathrm{keV}$ ). X-ray lines are produced also, in case of $\mathrm{W}$ anode, at $58 \mathrm{keV}\left(K_{\alpha}\right)$ and $67 \mathrm{keV}\left(K_{\beta}\right)$; since these represent $15 \%-20 \%$ of all the X-ray emitted photons, the spectra can be considered fairly continuous. The total number $\Phi$ of X-ray photons emitted is obtained by integrating the spectrum $N_{\mathrm{x}}(E)$, namely, photons at an energy $E$, over the X-ray energy range $E_{\text {min }}-E_{\text {max }}$ (Equation 1$) ; \Phi$ scales $^{3}$ as $Z \cdot\left(V_{\mathrm{p}}\right)^{2} \cdot I \cdot t$, where $Z$ is the atomic number of the anode, $I$ the electron current (in $\mathrm{mA}$ ) of the X-ray tube, and $t$ the exposure time (in seconds). The fluence $(\Phi / S$, where $S$ is the surface) is the number of photons per unit area $\left(\mathrm{ph} / \mathrm{mm}^{2}\right)$. The total X-ray energy W carried by the photons is obtained by integrating the spectrum $N_{\mathrm{x}}(E)$ multiplied by the photon energy $E$ (Equation 2). The energy fluence, or exposition, is $W / S\left(\mathrm{~J} / \mathrm{mm}^{2}\right)$; it scales ${ }^{3}$ as $Z \cdot\left(V_{\mathrm{p}}\right)^{5} \cdot I \cdot t$ and it is proportional to the absorbed dose.

$$
\begin{gathered}
\boldsymbol{\Phi}=\int_{E_{\min }}^{E_{\max }} N_{X}(E) d E \\
W=\int_{E_{\min }}^{E_{\max }} N_{X}(E) E d E
\end{gathered}
$$

The response of the detectors used so far in medical imaging is proportional to the energy fluence, while for new generation of C-MOS imagers in photon counting, the response is proportional to the number of photons, as we will discuss in the following sections.

X-ray photons interact with the target (subject) by means of absorption and scattering processes. Absorption occurs through the photoelectric effect, where the photon is absorbed by an inner shell electron of the atom and ejected (ionization). X-ray photon can also be scattered (see the work by Sprawls ${ }^{4}$ for an introductory description). Rayleigh scattering (elastic) is negligible in X-ray domain, while Compton scattering with the outer electrons is important, more and more at higher energies $(E>80 \mathrm{keV})$, where the photoelectric effect falls down, as shown in Table 1, for human body. See the book of Michette and Buckley ${ }^{5}$ for a detailed treatment of the physics of the X-ray interactions with the matter and the book of Bushberg et $\mathrm{al}^{3}$ for applications to medical imaging.

Since Compton effect is an inelastic scattering, the photon is deviated from its original direction and the energy is reduced, because part of it is released to the escaping electron. The X-ray photons contributing to the formation of the radiographic image on the detector are those transmitted (they do not interact with the target) and the scattered ones. Compton effect then is not a favorable process, because the change of direction of the photon degrades the spatial resolution, and its change of energy reduces the effectiveness of the energy resolution, because of overlapping of the bands.

Table I X-ray interaction with target as function of $k V_{p}$

\begin{tabular}{llll}
\hline $\begin{array}{l}\text { High voltage } \\
\left(\mathbf{k V}_{\mathbf{p}}\right)\end{array}$ & $\begin{array}{l}\text { Photoelectric } \\
(\%)\end{array}$ & $\begin{array}{l}\text { Compton } \\
(\%)\end{array}$ & $\begin{array}{l}\text { No interaction } \\
(\%)\end{array}$ \\
\hline 50 & 78 & 21 & 1 \\
80 & 46 & 52 & 2 \\
110 & 23 & 70 & 7 \\
\hline
\end{tabular}




\section{Detector parameters}

Imaging quality in radiography is due to three major contributions:

- Detectors (efficiency, noise, spatial resolution, dynamic range, contrast)

- Geometry (source-subject-detector distances, focal spot size, plane misalignment)

- Object (nature, atomic numbers of elements, shape)

We do not take into account here the geometrical factors because they are well known and do not depend on the different detection techniques. We focus on the detector parameters and then on the processes into the object.

The most relevant imaging quality parameters for an $\mathrm{X}$-ray detector are as follows ${ }^{6,7}$ :

- Spatial resolution

- Detection efficiency (DE)

- Sensitivity and dynamic range

- Contrast

- Noise

Spatial resolution is the smallest dimension by which spatial changes in the X-ray flux can be measured; it is a convolution of the physical processes occurring inside the detector: for digital detectors, for example, it depends on the conversion of X-ray photon, charge generation, drift, collection of charges, and signal pickup, and not simply on the pixel size. Spatial resolution is fully described by means of the modulation transfer function, ${ }^{8}$ and it is often quantified as lines $/ \mathrm{mm}$ that can be discriminated. $\mathrm{DE}$ is related to the probability of conversion of the X-ray photon and its transduction in a recorded signal; it indicates the number of photons that are required to generate a signal (response). Sensitivity is the change of the detector's response due to a change of the X-ray intensity arriving on it, while dynamic range, called also "latitude" for films, is the interval of intensity between minimum detection threshold and saturation. It is important to have a detector adequate to measure the entire range of intensity from the nonattenuated beam to the one passing through the densest and thickest part of the object (subject). Contrast is the ability to distinguish close structures with similar attenuation lengths (for example, liver-spleen, fat-muscle) or an object compared to background. A useful definition can be

$$
\mathrm{CR}=\left(I_{\mathrm{o}}-I_{\mathrm{b}}\right) /\left(I_{\mathrm{o}}+I_{\mathrm{b}}\right)
$$

where $I_{\mathrm{b}}$ is the intensity of the background and $I_{\mathrm{o}}$ the intensity of the object. Contrast is often also used to indicate the number of gray levels between black and white: at low contrast, there are more gray levels ("long scale"), edges are smoother, and less details can be detected, while at high contrast, there are lower levels of gray ("short scale") and sharper edges, and more details can be revealed. The relation between contrast and detail is often represented by means of the plot contrast versus object size, where the performance of a detector is represented as a curve, similar to an hyperbole branch, showing the contrast necessary to reveal an object of given size: of course, for small object size a larger contrast is required. Noise is the lowest level of detectability due to intrinsic uncertainties or fluctuations (such as granularity for film or electronic noise for digital detectors). In reality, detection is the measurement of a signal-to-noise ratio (SNR), ${ }^{9}$ namely, the signal compared to the level of noise. So the quality is not only due to the amplitude of the response (signal) alone, but its comparison with the noise has to be taken into account. Sometimes, DE and SNR are combined in the so-called quantum detection efficiency. ${ }^{10}$

Unfortunately, there is not a unique parameter to identify the imaging quality or the capability in detecting medically relevant details or other information. These physical parameters of a detector are very useful to describe its properties, but all of them contribute to the effective diagnostic capability. A detailed discussion of these subjects can be easily found in the literature. ${ }^{11-13}$

At lower $\mathrm{kV}_{\mathrm{p}}(60 \mathrm{kV})$, the subject contrast is higher because the mass absorption coefficient is more sensitive to the $\mathrm{X}$-ray energy and the tissue composition. Compton diffusion is lower, and the detectors also have a better contrast because DE is higher and spatial resolution is better. In general, the goal of radiography is the detection of something (bones, an organ, or part of it) compared to the background, thanks to a high contrast. In order to do it, lower $\mathrm{KV}_{\mathrm{p}}$ is preferable. At lower energy, absorption is higher, but the signal produced in the detector is lower, and so is the SNR. This is compensated by the increase of the quantity $I \cdot t$ (in $\mathrm{mAs}$ ), which implies, in turn, an increase of the patient absorbed dose. Higher values of $\mathrm{kV}_{\mathrm{p}}$ cause an increase of SNR, but because of Compton scattering, contrast is degraded and high-energy stray $\mathrm{X}$-rays are increased. With the new detectors working in photon counting, which will be discussed in the following text, thanks to their higher dynamic range, sensitivity, and lower noise, it will be possible to work at a lower $\mathrm{KV}_{\mathrm{p}}$, at which the subject and detector contrast is higher, without reducing the SNR.

\section{Photographic films}

For almost a century, X-ray radiographies were made with photographic films, based on gel with $\mathrm{AgBr}$ or $\mathrm{AgI}$ grains. ${ }^{14}$ 
The purely analogic response is the optical density (OD), namely, the blackening of the slab. The DE is low (about $1 \%$ of the incident $\mathrm{X}$ photons are detected), and its response (OD) is not linear with exposure, having a typical " $S$ " shape (Figure 1). At low ("foot") and high ("shoulder") exposures, sensitivity is very low (under- and overexposition); therefore, the effective dynamic range is two orders of magnitude (OD from 0.5 to 2.5 ) or slightly higher. Spatial resolution is very good (up to 100 lines/mm), and they have large area. Sometimes, image intensifiers with phosphors $\left(\mathrm{CaWO}_{4}\right.$, $\left.\mathrm{LaOBr}: \mathrm{Tb}, \mathrm{Gd}_{2} \mathrm{O}_{2} \mathrm{~S}: \mathrm{Tb}, \mathrm{Y}_{2} \mathrm{O}_{2} \mathrm{~S}: \mathrm{Tb}\right)$ are used in front of the film to increase the DE (up to 20\%); in this case, the X-ray conversion to visible light occurs in the phosphors.

Fluoroscopy, used for dynamic processes, has an image intensifier coupled with a video camera and a TV screen, making possible the visualization of radiography in real time. ${ }^{15} \mathrm{X}$-ray radiation is converted to visible light by a phosphor. Light extracts electrons from a photocathode, and electrons are accelerated by an electric field in a vacuum tube; they strike a phosphor and visible light is emitted and collected by a charge-coupled device (CCD). ${ }^{16}$ Intensifiers have enough spatial resolution, with good contrast and detail, to be very useful in cases such as angiography, for which time resolution is required.

\section{Imaging plates}

Nowadays, photographic films have been widely replaced by the imaging plates, also called storage phosphors

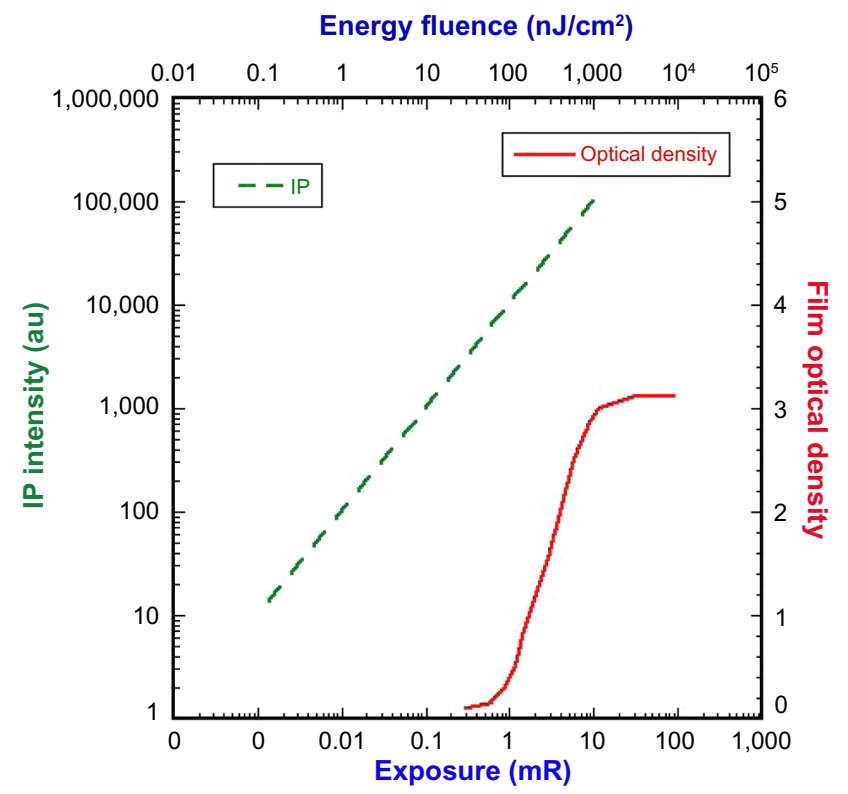

Figure I Response of film (red curve) and image plate (green curve) versus exposure (or energy fluence).

Abbreviations: au, arbitrary unit; IP, imaging plate.
(Barium fluorohalides BaFBr:Eu ${ }^{2+}, \mathrm{BaFCl}: \mathrm{Eu}^{2+}, \mathrm{RbBr}: \mathrm{Tl}+$, $\mathrm{CsBr}: \mathrm{Eu}^{2+}$ ), where X-rays absorbed by the material produce metastable states, and the plate is read out by recording the luminescence induced by a laser. ${ }^{17,18}$ They have DE similar to that of film, but the response with exposure is linear, and the dynamic range is almost four orders of magnitude (Figure 1). Despite the spatial resolution (5-15 lines/mm) being lower than that with the film, the linearity and the larger dynamic range increase significantly the contrast. In addition, imaging plates are reusable, they do not require chemicals unlike films, data storage is easier, and data transfer is also possible. The digital image allows also postprocess analysis, such as filtering (for noise reduction), rendering (for the reconstructions), windowing (for the change of intensity scales), zooming, edge sharpness, and so on.

\section{CCDs}

In other applications, X-rays are converted to visible light by means of scintillators; the light is collected by bundles of optical fibers, coupled at the end to a visible CCD camera for radiography scan. Since CCDs have limited area (a few squared centimeters), optical fibers provide a demagnification of the image (fiber-optic taper). CCDs have very high spatial resolution and higher dynamic range than do films and show linearity with exposure; unfortunately, they have small active area and require scintillators for the X-ray conversion that degrade the spatial resolution. ${ }^{19}$

\section{Flat panel detectors}

In the last two decades, the so-called flat panel detectors (FPD) have been developed to combine the advantages of having an electronic conversion of the radiation, as for CCDs, and high DE and large active area at the same time. Two approaches have been studied: indirect (such as CCDs having a scintillator as X-ray converter to visible light) and direct conversion.

Indirect FPD (Figure 2) are made by a slab of scintillator coupled to a two-dimensional squared matrix of photodiode array made with amorphous silicon (a-Si), which is a fairly good semiconductor; it requires low voltage for biasing and can be machined with large areas (up to about $40 \times 40 \mathrm{~cm}^{2}$ ). Electric charges produced by a photodiode array are collected and electronically processed by thin-film transistors (TFT), individually per each pixel. ${ }^{20}$ The indirect approach is well known, being exploited since many years. Integration in one compact detector was thus successfully achieved. Nevertheless, indirect conversion suffers from degradation of the spatial resolution (light in the scintillator is transversally spread, even 


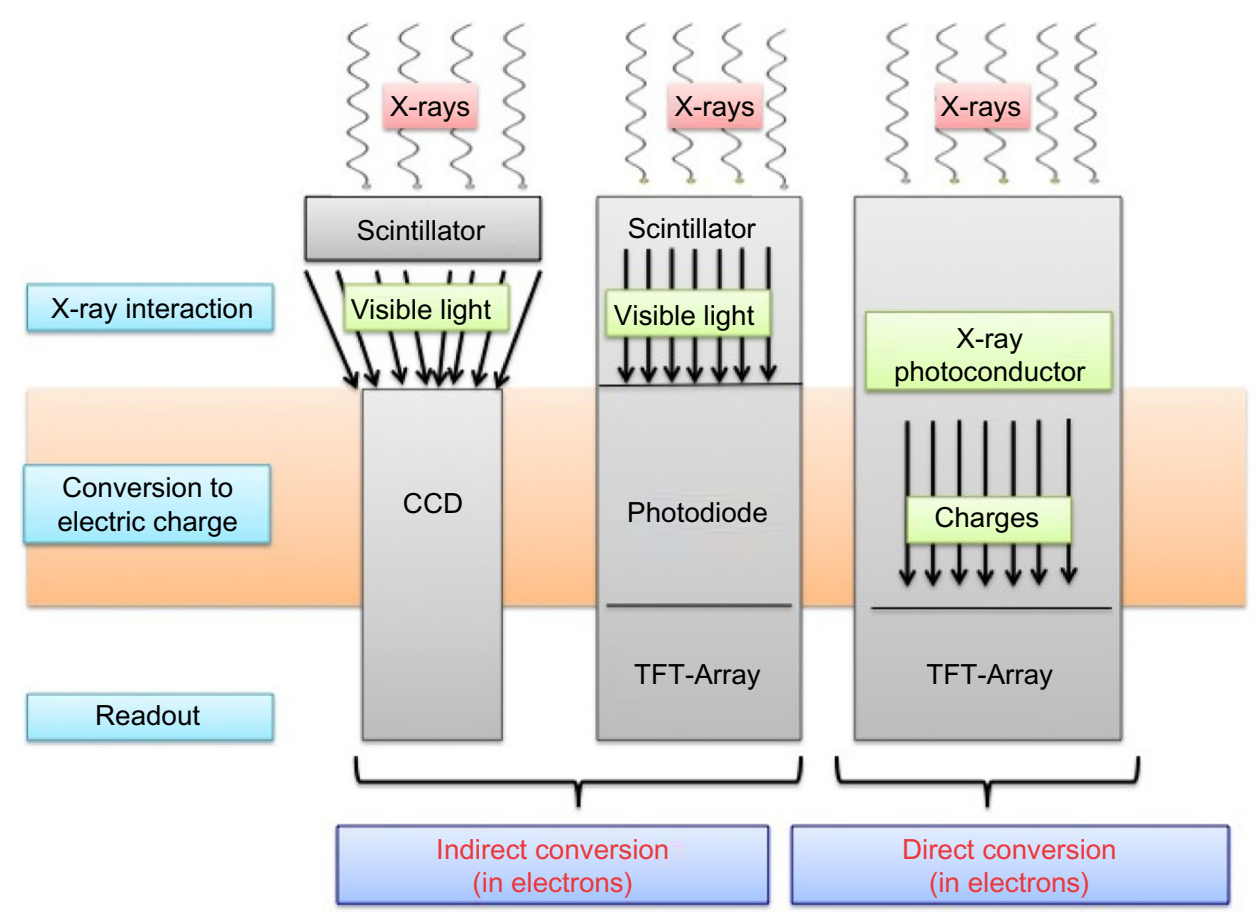

Figure 2 Direct and indirect conversion in charge-integration mode.

Abbreviations: CCD, charge-coupled device; TFT, thin-film transistor.

if columnar-shaped CsI scintillators have been used to reduce it), and the noise level of a-Si is pretty high. ${ }^{21}$

Direct FPD are made of a high $\mathrm{Z}$ semiconductor, amorphous selenium (a-Se), ${ }^{22}$ in which X-rays are converted directly to electrons that are then collected on a matrix of capacitor, coupled to a TFT for processing of the signal of each pixel (Figure 2). In principle, direct conversion is preferable, because of the higher spatial resolution and efficiency. Unfortunately, the DE of a-Se $(Z=34)$ is low at higher energy; so thick slabs are required to have enough DE, but it implies high voltages to be applied to the semiconductor to drift the charges to the pixels, which is risky for the TFT electronics. For this reason, it is used mainly for mammography. Attenuation coefficient (reciprocal of the attenuation length) is plotted in Figure 3, in the energy range of interest for medical imaging, for $\mathrm{Si}$, Se, photographic films, and CdTe, the last one being the most efficient for direct conversion. Better high $\mathrm{Z}$ semiconductors are under development, having higher $\mathrm{DE}$ and better dielectric properties, for example, CdTe, $\mathrm{CdS}, \mathrm{HgI}_{2}, \mathrm{PbI}_{2}, \mathrm{PBO}, \mathrm{TlBr}^{23,24}$ Unfortunately, they cannot be produced with large areas.

For these reasons, indirect FPD had a larger diffusion in these years, in radiography and computed tomography (CT). They have a pixel size of $\sim 100-200 \mu \mathrm{m}$, noise $\sim 1,000$ electrons, sensitivity $\sim 20 \mathrm{LSB} / 1 \mathrm{mR}$ (20 times more sensitive than film), dynamic range a few thousands, spatial resolution 5-10 lines/mm, digital output $12-14$ bits, and energy range 20-100 keV.

\section{Photon counting and C-MOS imagers}

All the detectors described in the previous section are "analog" in terms of X-ray photon detection, being "digital"

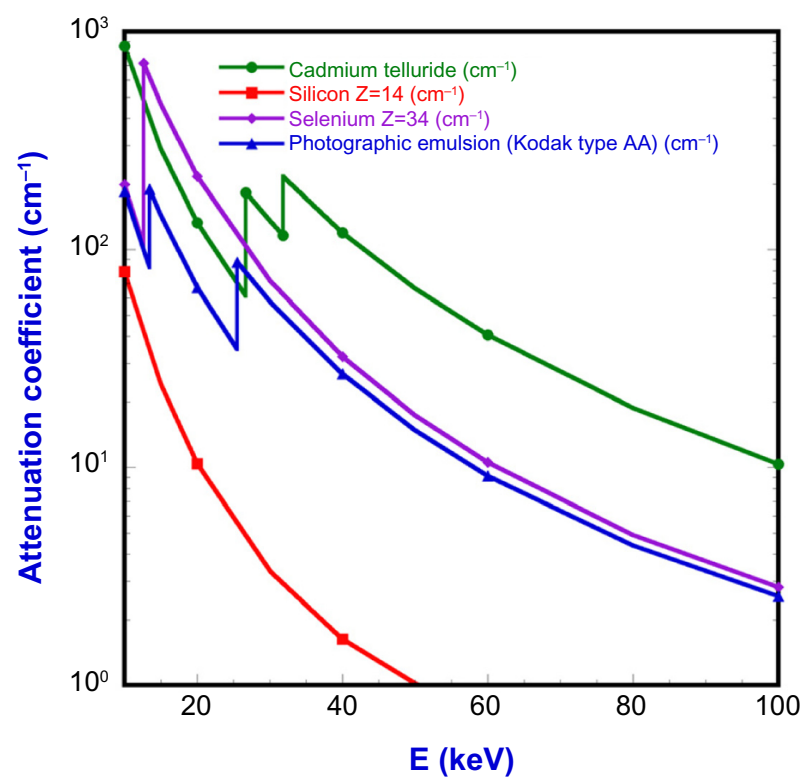

Figure 3 Attenuation coefficient for film, Se, Si, and CdTe. 
only in the way in which the converted information is spatially recorded in a squared matrix of pixels and the analog intensity is converted in a digital scale (in bits). Of course, films remain analog also in the sense of the data recording. X-ray photons convert to something else (blackened grains in film, metastable states in image plate, visible photons in scintillators, electron-ion pairs in a-Se) losing the information of their individuality and energy. The interaction of the X-ray beam with the subject to be imaged depends strongly on the X-ray energy, but this information cannot be exploited because it is lost in the detection. For example, one photon at $60 \mathrm{keV}$ could produce, in principle, the same signal as two photons of $30 \mathrm{keV}$ or three photons of $20 \mathrm{keV}$, while the transmission through the target of a photon of 60,30 , or $20 \mathrm{keV}$ is very different. In analogy with visible light, where the spectrum is divided into colors, we can claim that radiography has been done so far in black and white (false colors of computer images represent only a way of visualization), taking into account only intensity (gray levels) and not their energy ("colors").

New generations of X-ray detectors, solid-state C-MOS imagers ${ }^{25,26}$ or GEM (gas electron multipliers) gas detectors, ${ }^{27}$ developed in scientific research domains, opened the way for photon-counting imaging. In this approach, each photon is individually detected and its energy estimated. It offers three unprecedented advantages. First, it implies a general improvement of the physical parameters, on which imaging quality is based. Second, this is really a "digital" detection; so many data treatments can be done postprocess, for example, combinations of adjacent pixels (spatial binning) or time integration to increase the total signal or SNR or contrast. Third, it allows the energy discrimination in bands of the X-ray photons, opening the way to a "color X-ray radiography and/or tomography".

\section{Advantages of photon counting}

In the direct FPD a-Se detectors discussed in previous sections, the semiconductor, due to its limited purity (amorphous and not crystalline, because of the large area), is not depleted of free charges; so when a photon is converted, the limited number of electrons $\left(\mathrm{e}^{-}\right)$produced, $1 \mathrm{e}^{-}$per $3.6 \mathrm{eV}$ of photon energy, are partially hidden by the free charges. In addition, the lower performances of the semiconductor reduce the efficiency of the charge drift and collection on the pixel. The noise level associated with each pixel is about $1,000-2,000 \mathrm{e}^{-}$. As a consequence, a single photon cannot be detected.
In the C-MOS imagers, the semiconductor is fully depleted with a moderate electric field (bias voltage) and the charge is drifted and collected effectively on the pixel. The development of the microelectronics allowed the realization of applicationspecific integrated circuit (ASIC) with millions of electronic components per squared millimeter. This highly integrated electronics, coupled to very small pixel size $(\sim 50 \mu \mathrm{m})$, reduces the electronic noise to the level about $50 \mathrm{e}^{-}$root mean square (rms), making possible the detection of a single photon, even without cooling of the electronics. In Figure 4, one of these new generation of CdTe C-MOS imagers (Pixirad, courtesy of Pixirad Ltd, Pisa, Italy) is shown as an example, having an active area of $31 \times 25 \mathrm{~mm}^{2}$. Each pixel, $60 \times 60 \mu \mathrm{m}^{2}$, of the semiconductor sensor ( $650 \mu \mathrm{m}$ thick of CdTe) is bump-bonded to the corresponding channels of the chip (ASIC), which provides the analog and digital treatment of the signal. ${ }^{28}$ The X-ray photon is converted into the semiconductor and electric charges are collected on the pixels and transferred to the underneath ASIC. The C-MOS microchip performs the electronic treatment of the signal per each pixel: signal conversion of the total collected charges, pulse shaping, discrimination, counting, storage of the counts, and data transfer. Single-photon counting is accumulated in a 15-bit counter per pixel. The discriminator allows the electronic and detector noise to be cut off, and only photons whose signal overcomes the threshold are counted. These detectors are called "noise-free" because of this, and the only uncertainty is due to the statistical Poissonian fluctuation; so if we count $\mathrm{N}$ photons, the standard deviation $\sigma=\sqrt{ } \mathrm{N}$ and the relative error is $\sigma / \mathrm{N}=1 / \sqrt{ } \mathrm{N}$ (with 10,000 counts, the relative error is $1 \%$ ). The conversion of an X-ray photon in the semiconductor generates a number of electrons proportional to the photon energy. Thanks to the electronic linearity, the peak amplitude of the signal is proportional to the X-ray photon energy. The time scale for the drift of the charges in the sensor, their collection on the pixel, and the signal processing is $<100 \mathrm{~ns}$. Count rate per pixel can then occur at the rate of $\mathrm{MHz}$ (one million of counted photon per pixel per second) without significant photon pile-up. Since thresholds can be adjusted, it means that it is possible to count all the photons exceeding the energy corresponding to the threshold. This is the fundamental feature for the energy-discrimination imaging. The high count rate and the depth of the bits allow a huge dynamic range (six orders of magnitude). Once all the thresholds are set to cut the noise off, the detector is able to count even a single photon over the full active area. Automatic procedures for the ASIC are established to equalize each pixel individually, compensating its different gain and threshold. 


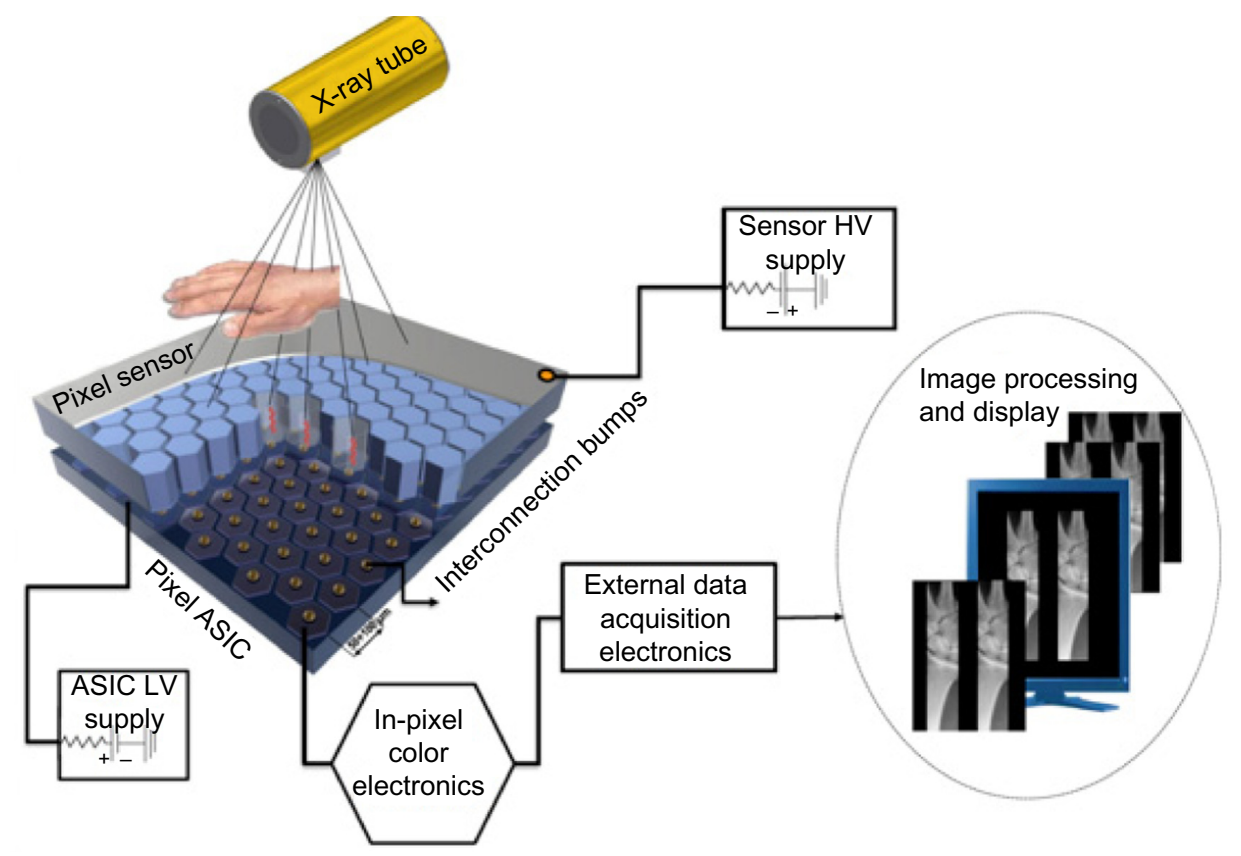

Figure 4 Schematic principle of working of a C-MOS imager.

Note: Reproduced from PiXirad. Image sensor: principle of operation of pixirad. Available from: http://www.pixirad.com/?page_id=2. Accessed December 22, 20I4. ${ }^{50}$ Abbreviation: ASIC, application-specific integrated circuit; LV, low voltage; HV, high voltage, C-MOS, complementary metal-oxide-semiconductor.

Since the detector has hundreds of thousands of pixels, readout time is of the order of $10 \mathrm{~ms} /$ frame. Since CdTe sensor is a high $Z$ (atomic number) semiconductor and its density is pretty high $\left(5.85 \mathrm{~g} / \mathrm{cm}^{2}\right)$, its DE is high in the whole range $2-100 \mathrm{keV}$, as can be seen in Figure 3. Silicon and selenium cannot be used for direct conversion because their DE falls down respectively at 30 and $60 \mathrm{keV}$.

In Table 2, the order of magnitude of the minimumdetectable X-ray fluence $\left(\mathrm{ph} / \mathrm{mm}^{2}\right)$ is given for the detectors discussed so far, stemming from the combination of DE and noise level. Devices working in photon counting (noise-free) have virtually no detection threshold $\left(1 \mathrm{ph} / \mathrm{mm}^{2}\right)$.

Sensitivities also increase progressively from film to C-MOS imagers, even if at a lower rate (roughly one order of magnitude instead of many). A dose reduction is also associated with an increase of sensitivity, even if the correlation is not straightforward because dose depends also on the other parameters.

Table 2 Detection threshold

\begin{tabular}{ll}
\hline Detector & $\begin{array}{l}\text { Minimum detectable } \\
\text { fluence }\left(\mathbf{p h} / \mathbf{m m}^{2}\right)\end{array}$ \\
\hline Film & $10^{6}$ \\
Imaging plate & $10^{4}$ \\
Flat panel detector & $10^{4}$ \\
C-MOS imager & 1 \\
\hline
\end{tabular}

Abbreviation: C-MOS, complementary metal-oxide-semiconductor.
A large effort has been undertaken in the last 10 years for developing these C-MOS imagers, promoting large collaborations between universities, national laboratories, and international institutions, such as in the case of Medipix detector ${ }^{29}$ It is formed by a square matrix of $256 \times 256,55 \times 55$ $\mu \mathrm{m}^{2}$ pixels, resulting in an active area of $1.4 \times 1.4 \mathrm{~cm}^{2}$, with a flexible ASIC that can work both in photon-counting and in charge-integration mode.

See the technical report of Taylor ${ }^{16}$ for a description of architecture of CCD and C-MOS imaging arrays. In general, C-MOS imagers have high efficiency (direct conversion, crystalline semiconductor, low noise, and high sensitivity of the electronic front-end in the ASIC), are noise-free, and have high dynamic range and spatial resolution. All these nice features mean for medical imaging a reduction of dose and an increase of contrast. The detector response (peak amplitude) is proportional to the X-ray photon energy, linear up to a few tens of $\mathrm{keV}$ and then a lack of linearity due to the electronic nonlinearity and multiple counting when charge is collected by more than one pixel. This effect is due to the fact that high-energy photons release energy (and then electrons) over a larger volume and the charge is not collected only in one pixel, but a small fraction arrives on a neighboring pixel. This effect degrades the spatial resolution and implies an underestimation of the $\mathrm{X}$ energy. ${ }^{32,33}$ Smart techniques in the ASIC architecture are under development to limit or correct this effect. At high-energy K-edge, escaping 
$X$-rays (produced by the radiative deexcitation of an inner shell of the high $\mathrm{Z}$ semiconductor following the photoelectric absorption) have to be taken into account, causing spatial degradation and energy underestimation. Detection in photon-counting mode allows also the correction of the $\mathrm{DE}$ at all the energies.

Unfortunately, for the moment, these detectors have a limitation in the active area (tens of $\mathrm{cm}^{2}$ ). These detectors are built to be side buttable, so a $2 \times 2$ squared array can be formed $\left(5 \times 5 \mathrm{~cm}^{2}\right)$ or an $8 \times 1$ linear array $\left(25 \times 2.5 \mathrm{~cm}^{2}\right)$. These assemblies can be used for $\mathrm{CT}$ or for scanning radiography. In Figure 5, an example of a radiograph of a rat's chest is shown (courtesy of Pixirad, Ltd).

\section{Energy-resolved imaging}

Dual-energy imaging is based on the acquisition of two distinct images taken in different spectral ranges. ${ }^{34}$ This is usually carried out in two ways: dual-kV and dual-detector layer techniques. In the first case, the X-ray tube is switched at two different $\mathrm{kVp}$, one lower and the higher one with suitable filters to cut the low-energy side. For example, one at $70 \mathrm{kV}$ and the other one at $140 \mathrm{kV}$ with a tin filter, or $60 \mathrm{kV}$ with samarium and $90 \mathrm{kV}$ with copper, or $75 \mathrm{kV}$ with holmium and $140 \mathrm{kV}$ with copper. ${ }^{35}$ This solution, based on two acquisitions, is unfortunately sensitive to the patient's motion.

Alternatively, for tomography only, two X-ray tubes are used, set at two different voltages and placed in an orthogonal configuration. The other technique is based on dual-layer detector. In this case, the spectrum is fixed and the detector

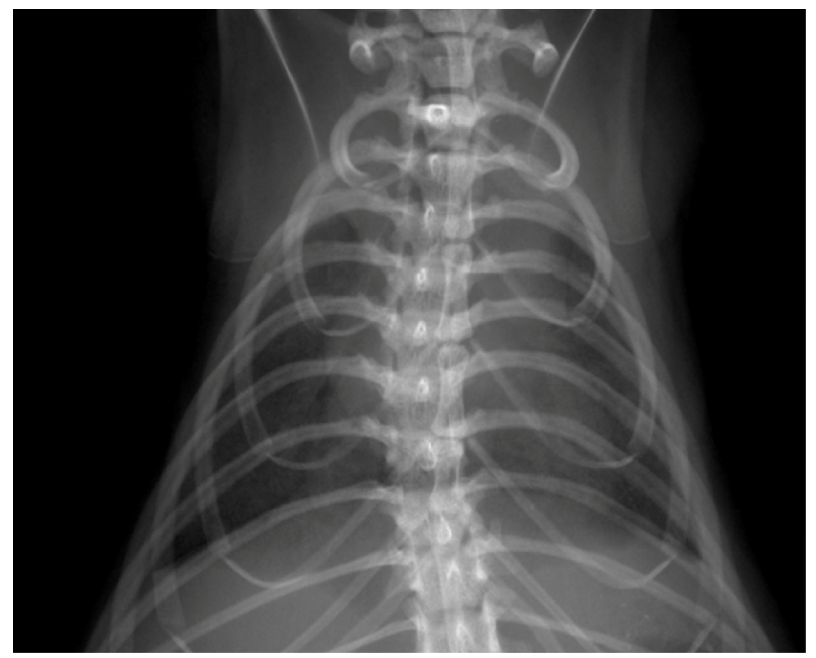

Figure 5 Digital image of a rat's thoracic cavity.

Notes: The image was obtained with the Pixirad C-MOS imager. Heart and lungs are visible. Reproduced from PiXirad. Digital image of a rat's thoracic cavity. Available from: http://www.pixirad.com/?page_id=396. Accessed December 22, 20I4. ${ }^{51}$ Abbreviation: C-MOS, complementary metal-oxide-semiconductor. should discriminate the photons in two energy bands. This is accomplished with two stacked scintillators separated by a filter, with photomultipliers aside. ${ }^{36}$ The first scintillator detects low energies, while the second one only the more energetic X-rays. This solution suffers from a poor spectral separation, and it requires a dedicated detection system. The new generation of photon-counting detectors offers a better energy discrimination. Dual-energy imaging is useful when two different materials are present in the target. For example, in medical imaging it is useful when the body can be considered a sort of a binary system, as bones and soft tissues in bone densitometry, or in functional imaging, where a contrast agent has to be imaged. Dual-energy X-ray imaging is now a reality in more than one application. This technique is used in dual-energy X-ray absorptiometry (DXA) for bone mineral density and body composition assessment, ${ }^{37,38}$ in chest radiography, for finding low-contrast nodules as an indication for lung cancer, ${ }^{39}$ in dual-energy contrast-enhanced digital subtraction mammography ${ }^{40}$ or for metal artifacts in radiology.

Dual energy is used also in connection with molecular markers, to target a given organ or to follow dynamical processes, such as blood fluxes or heart activity. Iodine, gadolinium, and barium are mostly used in these markers, because of their K-edge transitions falling in a useful part of the X-ray spectrum. Gadolinium has the $K_{\alpha}$ line at $43 \mathrm{keV}$, iodine at $28.6 \mathrm{keV}$, and barium at $32.2 \mathrm{keV}$. By acquiring two images, one for X-ray energies below the absorption edge and the other one above, the marker can be easily imaged by difference. This technique is applied for coronary angiography, iodine-enhanced imaging for myocardial infarctions, liver tumors or other inner organ tumors, and so on.

Multienergy is an extension of the method, potentially useful for targets made of many materials, but it is extremely challenging. ${ }^{41}$ There are systems increasing the level of spectral emissions of the X-ray tube, in subsequent exposures, but the device is difficult to tune and the maintenance is complex. The only way toward a real multienergy system is the development of detectors able to discriminate in different energy bands, being impossible to produce many separated spectral ranges using X-ray tubes, even neglecting the problem of misregistration of many successive exposures. At present, multienergy CT is still at the research level or preclinical investigations. New photon-counting detectors, such as the C-MOS imagers with CdTe, have small area, but they can be easily coupled to form a one-dimensional detector for CT scanning. One application is the imaging of multiple contrast agents, by means of their K-edges. Next to 
the traditional ones, such as iodine, gadolinium, and barium, high $\mathrm{Z}$ elements, such as gold and platinum, are being investigated to be coupled to functional nanoparticles targeting specific enzymes and cells. Multienergy CT should also improve the morphological imaging, thanks to the intrinsic tissue contrast. ${ }^{42}$

If dual energy is powerful to image a molecular marker (as in angiography) or enhance the contrast of a part compared to the background, multiple-energy X-ray imaging opens the way to detect the nature of the materials present inside the target. This is possible only by measuring the selective absorption at the different energy bands. The transmission $I / I_{\mathrm{o}}$ of an X-ray beam of energy $E$ and intensity $I_{\mathrm{o}}$ entering in a homogeneous target of thickness $t(\mathrm{~cm})$ with a given composition results in:

$$
\frac{I}{I_{O}}=e^{-\alpha(E) \rho t}
$$

where $I$ is the intensity at the exit of the target, $\alpha$ is the mass absorption coefficient of the material $\left(\mathrm{cm}^{2} / \mathrm{g}\right)$, and $\rho$ is its density $\left(\mathrm{g} / \mathrm{cm}^{3}\right)$. In Figure 6 the attenuation coefficients $\alpha \rho$ are plotted for a few parts or elements of the human body, showing a strong dependence on the photon energy $E$. Calcium and bones are by far more opaque than other tissues. Integrating in the whole energy range, as in the standard radiography and tomography, bones can be easily recognized, but practically there is almost no difference between the other soft tissues. If we zoom on the low-energy range 20-40 keV and we perform a scan in energy, different soft tissues (adipose, breast, muscle) can be discriminated, at least in principle. In these energy ranges, the $\alpha \rho$ coefficient is higher and consequently also the absorption. Consequently, the detector is required to have a very high dynamic range, together with energy-discrimination capability: This is exactly the case for C-MOS imagers in photon counting. Multienergy discrimination is extremely sensitive on the chemical composition of the material. A seminal work has been done in 2003 by Pacella et al ${ }^{43,44}$ in developing X-ray imaging detectors based on gas electron multipliers, with energy resolution capability. Pacella et $\mathrm{al}^{45}$ demonstrated in 2006 that energy-resolved imaging is a powerful way to distinguish materials that cannot be discriminated with the standard (energy integrated) $\mathrm{X}$-ray imaging, and even more, it could reveal the nature of the material despite the poor energy resolution of the detector.

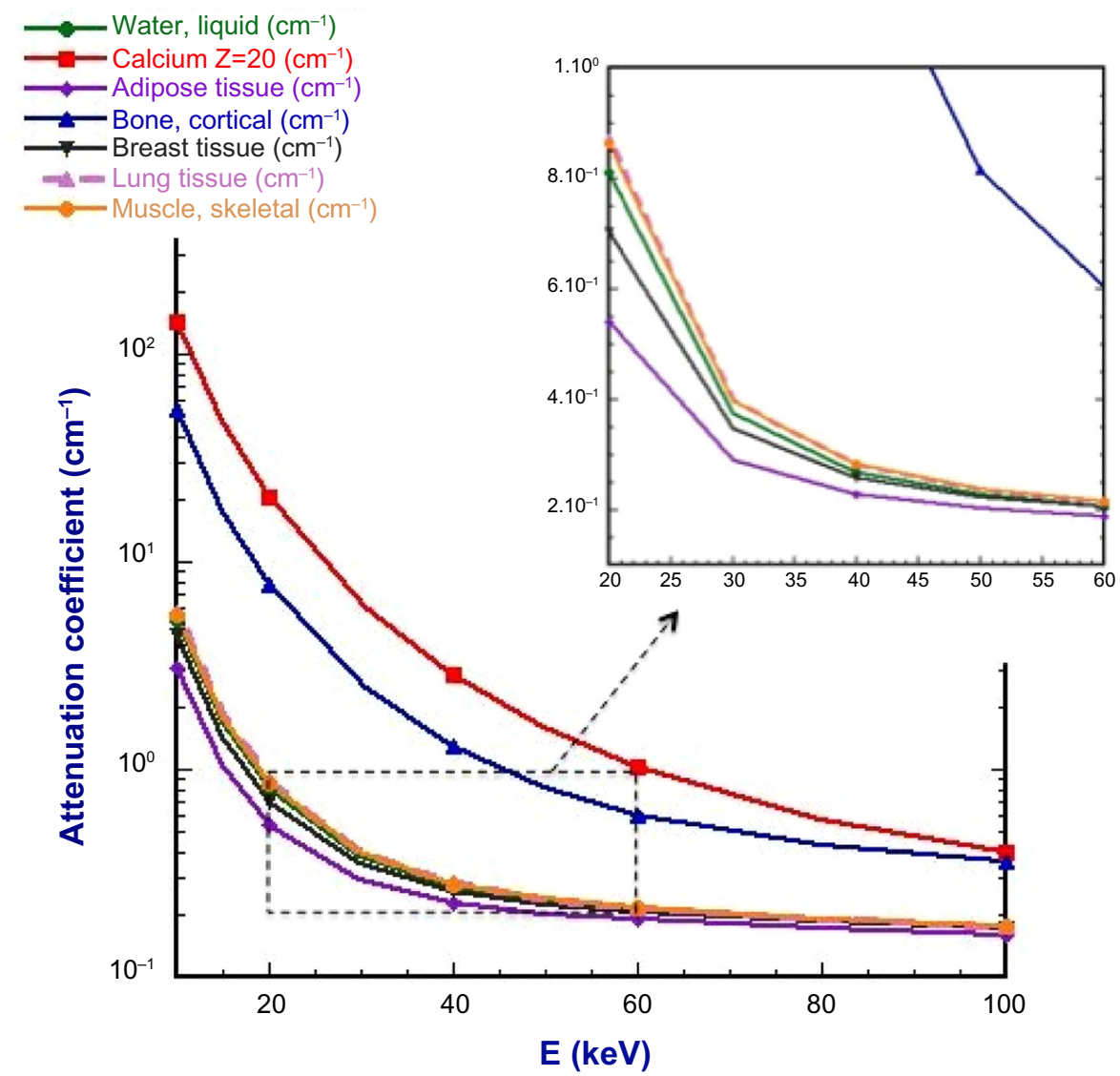

Figure 6 Attenuation coefficients $\alpha \rho$ for a few parts or elements of the human body. 
The energy range (2-15 keV) was divided in 25 energy bands and a scan of threshold was performed. The spectrum of the absorbed radiation in these bands, for different samples (fat, plastic tape, $\mathrm{CaCl}$ ), was estimated and the transmission coefficients were derived (Figure 7). Despite the poor energy resolution, absorption edges of $\mathrm{Ca}, \mathrm{P}$, and $\mathrm{Cl}$ could be reconstructed (Figure 7). Even if the work has been done in a low-energy interval $(2-15 \mathrm{keV})$ and with a GEM gas detector, it represents a proof of principle of the attractiveness of the so-called multicolor X-ray imaging.

Multienergy is much more challenging because the finite energy resolution of the detector overlaps the results in the different bands and because the spectrum at lower energies is distorted by the effect of charge sharing following the detection of high-energy photons. Solutions will be investigated both on the hardware side, improving the ASIC architecture to tackle and compensate the charge sharing, and on the software side for the finite resolution effects. Moving at higher energies, the probability of photoelectric effect decreases, while the Compton scatter increases, producing a degradation of the spatial resolution and a further overlapping of the band spectra. The energy discrimination in bands of a CdTe C-MOS imager (Pixirad) has been studied by the author using soft X-ray (SXR) line transitions generated by fluorescence on different samples $(\mathrm{Ca}, \mathrm{Fe}, \mathrm{Cu}, \mathrm{Br}, \mathrm{Mo}, \mathrm{Ag}$, $\mathrm{I}, \mathrm{Ta})$ and by means of $\mathrm{BaCs}$ radioactive source. A scan of threshold, common for all the pixels, has been performed for each X-ray line and plotted in Figure 8. Scans are normalized to the value corresponding to the minimum threshold ( $\left.350 \mathrm{e}^{-}\right)$. The threshold is defined as the number of electrons collected on the pixel (and then transformed in a pulse), varying

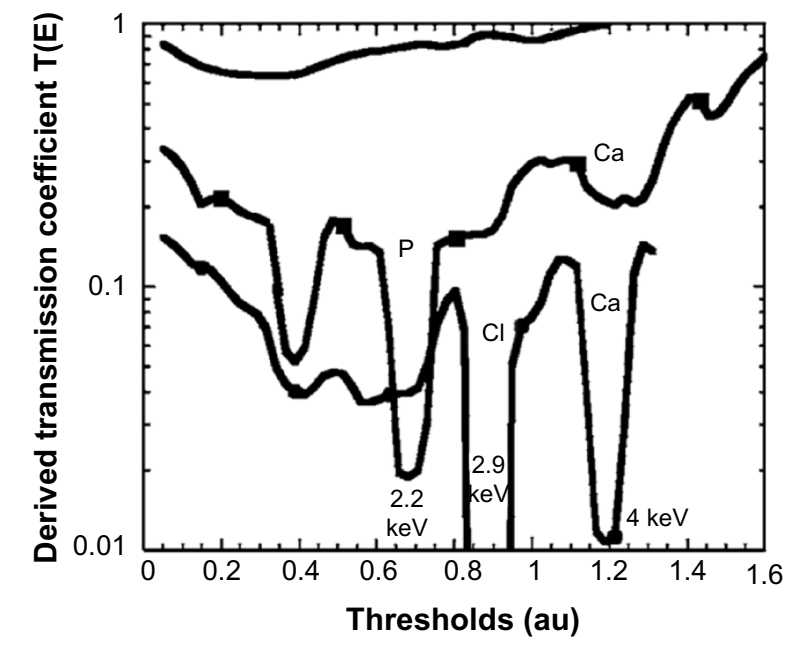

Figure 7 Derived transmission coefficients, respectively from the top, for plastic tape, fat, and $\mathrm{CaCl}$. Absorption edges of $\mathrm{P}, \mathrm{Ca}$, and $\mathrm{Cl}$ can be recognized. Abbreviation: au, arbitrary unit.

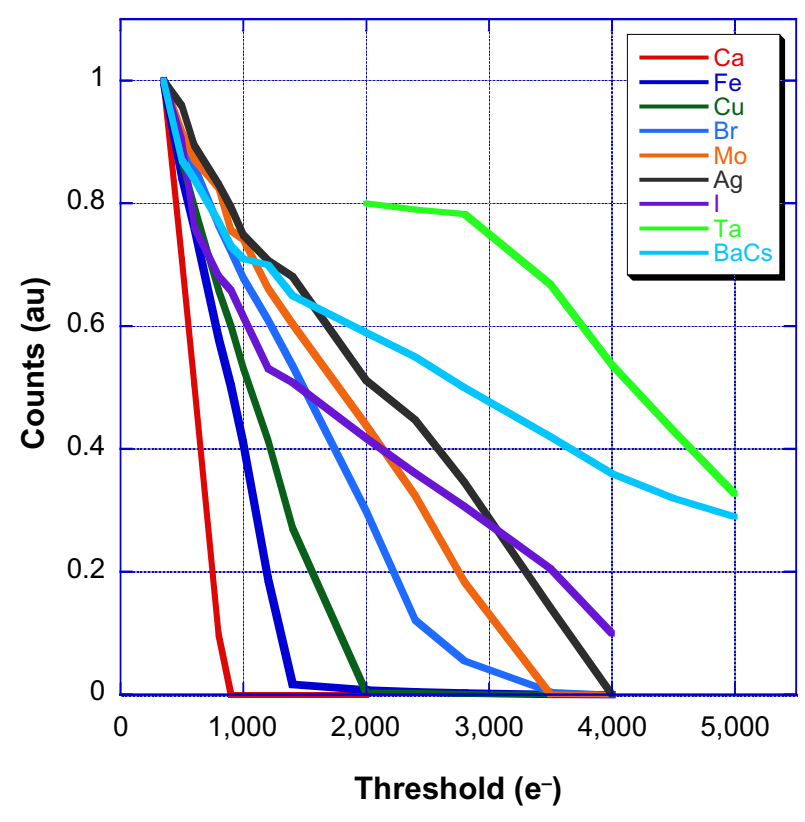

Figure $\mathbf{8}$ Threshold scan of the normalized intensity for monochromatic X-ray lines. Abbreviation: au, arbitrary unit.

from 50 to 5,000 electrons, while the dynamic range of the preamplifier is wider. The scans start from 350 electrons in order to cut off the noise completely. At fixed threshold, X-ray energies below the value corresponding to the threshold are cut; energies around the region of the threshold are partially cut, while energies well beyond the threshold are unaffected. This is a sort of "smooth" discrimination, due to the poor spectral resolution of the detector. The correspondence between threshold and X-ray energy $E$ for cutting completely the photons of energy $E$ (Th0) or $50 \%$ of them (Th50) is

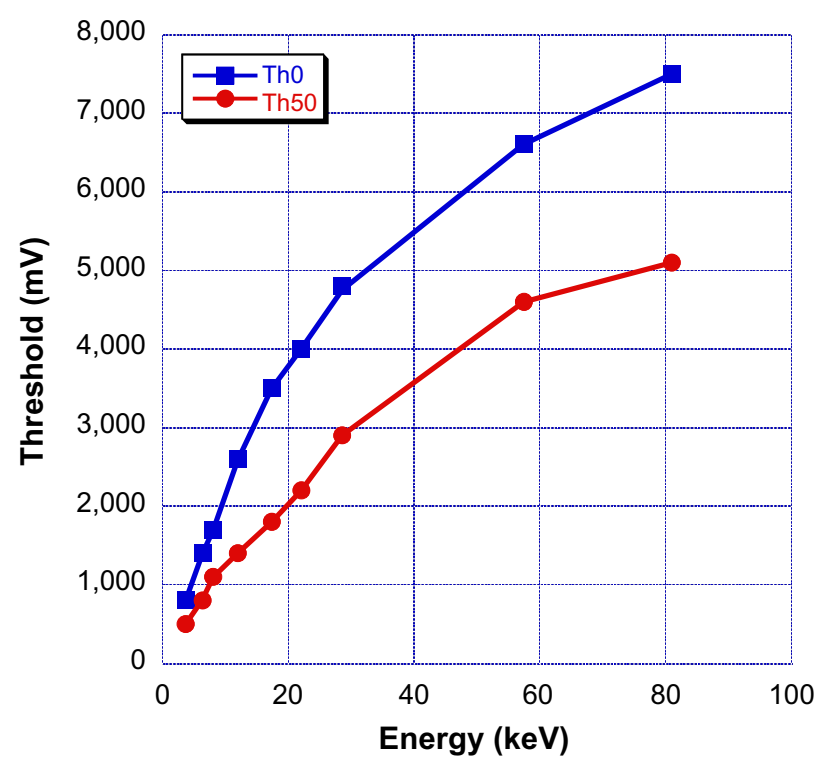

Figure 9 Correspondence between threshold and X-ray energy $E$ for cutting completely the photons of energy $E$ (Th0) or $50 \%$ of them (Th50). 
plotted in Figure 9. These curves are linear up to about 30 $\mathrm{keV}$, beyond which they continue to be proportional, even if not strictly linear, up to $80 \mathrm{keV}$. The lack of linearity is due to the electronic nonlinearity of the preamplifiers and the onset of multiple counts.

Nowadays, a lot of research work is in progress to study this multiband imaging, in the right energy intervals (20-120 keV) for medical applications, by means of C-MOS imagers, mainly with CdTe sensors, but also with cadmium zinc telluride (CZT), mercuric iodide $\left(\mathrm{HgI}_{2}\right)$, and gallium arsenide (GaAs). Energy-resolved photon-counting detectors are able to measure spectra from polychromatic sources, like a standard X-ray tube from 60 to $120 \mathrm{KV}_{\mathrm{p}}$, resolve absorption edges of $\mathrm{Gd}$ and $\mathrm{I}^{46}$, and identify different materials in tomographic imaging, based on the energy dependence of the linear attenuation coefficient $(\alpha \rho)$.

Discussions about the possibilities offered by photoncounting detectors with energy resolution, in medical X-ray CT and imaging, can be found in the work of Butler et al, ${ }^{47}$ where the improvements of Medipix-3ASIC, with multiple energy thresholds, will allow contemporary information on spatial, time, and spectral distribution of the X-ray beam. Techniques for correcting the spectral distortions due to the charge sharing are described by Tlustos, ${ }^{48}$ together with the imaging capabilities with four energy beams and molecular markers of I (for blood) and Ba (for lung). A review paper has been published recently (2013) by Taguchi and Iwanc$\mathrm{zyk}^{49}$ discussing in detail the performances of these photoncounting detectors with energy discrimination, including the strategies for reducing the distortive effects and showing the improvements in contrast capabilities, dose reduction, and spatial resolution for CT and X-ray imaging. Owing to energy discrimination in bands, simultaneous multiagent (with I, Ga, $\mathrm{Au}$, and $\mathrm{Bi}$ ) imaging for different functionalities becomes now possible, and nanoparticle contrast agents with heavy atoms ( $\mathrm{Ga}, \mathrm{Au}, \mathrm{Bi})$ are under investigation, offering a larger SNR, because of their higher energy $\mathrm{K}$ absorption edge.

\section{Future developments}

This section envisages possible lines of developments for the long-term future.

Following what was discussed in the previous section, in particular with the perspectives opened by the band energy discrimination, it appears evident that improvement of the X-ray radiographic scheme requires a better matching between object (patient), spectrum, and detector. These three components have to be considered in an integrated view, together with their dependence on energy. Optimization starts from the object, defining the most sensitive energy bands to probe it, maximizing the contrast, and minimizing the dose. Since mass absorption coefficients of the different tissues and organs vary widely in the whole energy range, in principle the X-ray spectrum should be tailored in each energy band to maximize the contrast and detail capability, taking into account the detector features. Working at lower energy can be fruitful because the mass absorption coefficients are more sensitive to energy (high derivative of $\alpha$ versus $E$ ). There are recent research works aiming to optimize the spectrum by means of filters, such as tin or other elements. Tailoring the spectrum is indeed extremely limited. The shape of the spectra emitted by the tubes is quite stiff, depending on the anode material (W mainly, or Mo, Ag) and high-voltage $\mathrm{KV}_{\mathrm{p}}$. If more energetic X-ray photons are required because of the opacity of the target, most of the photons emitted at lower energies are pretty useless, but they nevertheless contribute enormously to the absorbed dose.

A tremendous jump forward should be represented by a passage to a discrete set of X-ray lines instead of a continuous spectrum. If we could select a number of lines (5-10) in the energy bands optimized for the object to be studied, choosing also intensities separately for each one, we could strongly enhance the probing capabilities, minimizing the contemporary dose. X-ray radiography or tomography would be improved also because the effects of poor spectral resolution of the detector would be overcome, provided that separation of the lines is higher than the broadening effects of the detector. In this case, energy resolution would not be given anymore by the detector but by the intrinsic width of the generated lines that is much higher. In the case of continuous spectra, the transmitted spectrum is even more smoothed because of the photons scattered due to the Compton effect, thus reducing the energy sensitivity. This effect also is reduced in case of discrete spectrum. X-ray lines could be produced by fluorescence of an X-ray beam (broad spectrum) on specific targets or by means of Bragg diffraction of X-rays on crystals or on multilayer mirrors. At present, this is not viable at the level of $\mathrm{X}$-ray radiation exposures used in medical applications, because both the processes are highly inefficient $\left(\sim 10^{-3}\right)$. Alternatively, synchrotrons could provide line emissions with enough brightness, but they are big, very expensive facilities and they cannot be used in clinical practice. In the future, X-ray optics could be useful for collecting X-ray radiation emitted by the tubes, focusing it on the fluorescence targets, and collecting also the radiation produced by the target itself. X-ray optics based on polycapillary lenses are now used for laboratory experiments in 
which focusing of X-ray radiation is required. They are made of a bundle of hundreds thousands microglass tubes, each having a diameter of about $10 \mu \mathrm{m}$, squeezed at the extremes to provide the right curvatures to act as a focusing lens, with a few degrees of convergence (divergence). X-rays, entering into each glass fiber at grazing incidence, are guided to the exit through multiple reflections. These lenses have a cross diameter of a few millimeters, length of about $10 \mathrm{~cm}$, and input and output focal distance of a few centimeters. These polycapillary lenses have been recently characterized ${ }^{50}$ in terms of imaging properties and radiation collection/transport at large distances (up to $7 \mathrm{~m}$ ). This optics could play a role in the future for scanning radiography or tomography, to collect more radiation from the sources, transporting it at larger distances or combining X-ray beams coming from different sources. Another alternative in the future could be represented by the X-ray lasers as sources of bright and coherent monochromatic lines. ${ }^{51}$

Nowadays, discrete spectra are not an option, just personal ideas to envisage the future. On the other hand, multienergy imaging, even though it is at the very first steps, is already disclosing huge diagnostic potentialities.

\section{Conclusion}

A new generation of X-ray detectors, coming from scientific research and exploiting the progress of microelectronics, has been described. These C-MOS imagers have been compared with the detectors used in medical imaging: photographic film, imaging plate, and FPD. In terms of performances (DE, sensitivity, dynamic range, spatial resolution), they represent a real jump forward, and compared with flat panels, they can work in photon-counting mode. Because of this, they have only statistical noise (noise-free), a much larger SNR, and a wider dynamic range, and since they are intrinsically digital even in the detection, all the imaging data treatment can be applied postprocess without lack of information. Unfortunately, they have small active area; so they could be conceived in the future for scanning radiography or tomography. C-MOS imagers also open the perspective of energy-resolved X-ray imaging. This is a new emerging domain, rich of potentialities. Since the X-ray absorption coefficients of the different parts or organs of the patient (object) are strongly dependent on the X-ray photon energy, this multienergy ("colored") X-ray imaging could increase enormously the probing capabilities. Based on this new technique of color X-ray imaging, the entire scheme of source-object-detector could be revised in the future, optimizing the spectrum and detector to the nature and composition of the target to be investigated. In this view, a transition to a set of monoenergetic X-ray lines, suitably chosen in energy and intensity, could be envisaged, instead of the present continuous spectra.

\section{Disclosure}

The author reports no conflicts of interest in this work.

\section{References}

1. Linton OW. Medical Applications of X-Rays. Available from: http:// www.slac.stanford.edu/pubs/beamline/25/2/25-2-linton.pdf. Accessed November 12, 2014.

2. Sauerschnig M, Schaefer M. Industrial X-ray generation: new technology for expanding applications. 18th World Conference on Nondestructive Testing; April 16-20, 2012; Durban, South Africa.

3. Bushberg JT, Seibert JA, Leidholdt EM, Boone JM. The Essential Physics of Medical Imaging. 3rd ed. Philadelphia, PA: Lippincott Williams and Wilkins; 2012.

4. Sprawls P. Interaction of Radiation with Matter. Available from: http:// www.sprawls.org/ppmi2/INTERACT/\#CHAPTER\%20CONTENTS. Accessed November 12, 2014.

5. Michette AG, Buckley CJ. X-Ray Science and Technology. 1st ed. Bristol, UK: Institute of Physics; 1993.

6. Marshall NW. A comparison between objective and subjective image quality measurements for a full field digital mammography system. Phys Med Biol. 2006;51:2441-2463.

7. Borasi G, Samei E, Bertolini M, Nitrosi A, Tassoni D. Contrast-detail analysis of three flat panel detectors for digital radiography. Med Phys. 2006;33:1707-1719.

8. Carton AK, Vandenbroucke D, Struye L, et al. Validation of MTF measurement for digital mammography quality control. Med Phys. 2005;32:1684-1695.

9. Tapiovaara MJ. SNR and noise measurements for medical imaging, II: application to fluoroscopic x-ray equipment. Phys Med Biol. 1993;38:1761-1788.

10. Neitzel U. Determination of the detective quantum efficiency of a digital $\mathrm{x}$-ray detector: comparison of three evaluations using a common image data set. Med Phys. 2004;31:2205-2211.

11. Shepard CT. Radiographic Image Production and Manipulation. New York, NY: McGraw Hill; 2002.

12. Suetens P. Fundamentals of Medical Imaging. 2nd ed. New York, NY: Cambridge University Press; 2009.

13. Aichinger H, Dierker J, Joite-Barfuß S, Säbel M. Radiation Exposure and Image Quality in X-Ray Diagnostic Radiology. 2nd ed. New York, NY: Springer-Verlag; 2012.

14. Fujifilm. The Fundamentals of Industrial Radiography: Fujifilm Technical Handbook. Tokyo, Japan: Fujifilm Corporation; 1990. Available from: http://www.fujifilmusa.com/shared/bin/ix-film_ fundamentals_of_industrial_radiography.pdf. Accessed November 12, 2014.

15. Nickoloff EL. AAPM/RSNA physics tutorial for residents: physics of flat-panel fluoroscopy systems: Survey of modern fluoroscopy imaging: flat-panel detectors versus image intensifiers and more. Radiographics. 2011;31(2):591-602.

16. Taylor SA. CCD and CMOS Imaging Array Technologies: Technology Review (Technical Report EPC-1998-106). Cambridge, UK: Xerox limited; 1998. Available from: http://research.microsoft.com/ pubs/80353/CCD.pdf. Accessed November 12, 2014.

17. American Association of Physicist in Medicine. Acceptance Testing and Quality Control of Photostimulable Storage Phosphor Imaging Systems (AAPM Report No 93). College Park, MD: AAPM; 2006.

18. Leblans P, Vandenbroucke D, Willems P. Storage phosphors for medical imaging. Materials. 2011;4:1034-1086. 
19. Evans DS, Workman A, Payne M. A comparison of the imaging properties of CCD-based devices used for small field digital mammography. Phys Med Biol. 2002;47:117-135.

20. Medical Expo. The Online Medical Devices Exhibition. Available from: http://www.medicalexpo.com/medical-manufacturer/flat-paneldetector3133.html. Accessed November 12, 2014.

21. Samei E, Flynn MJ. An experimental comparison of detector performance for direct and indirect digital radiography systems. Med Phys. 2003;30:608-622.

22. Zhao W, Ji WG, Debrie A, Rowlands JA. Imaging performance of amorphous selenium based flat-panel detectors for digital mammography: characterization of a small area prototype detector. Med Phys. 2003;30:254-263.

23. Dendooven P. Detection of energetic particles and gamma rays semiconductor detectors. Available from: http://www.astro.rug nl/ peletier/Bas\%20Det\%20Tech\%202009\%20semiconductors.pdf. Accessed November 12, 2014.

24. Tanaka T. Development of High-Z Semiconductor Detectors and Their Applications to X-ray/gamma-ray Astronomy. Available from: https://www-group.slac.stanford.edu/ais/publicDocs/presentation77. pdf. Accessed November 12, 2014.

25. Bellazzini R, Spandrea G, Minutia M, et al. Direct reading of charge multipliers with a self-triggering CMOS analog chip with $105 \mathrm{k}$ pixels at $50 \mu \mathrm{m}$ pitch (physics/0604114). Nucl Instrum Methods A. 2006;566:552-562.

26. Bellazzini R, Spandre G, Brez A, Minuti M, Pinchera M, Mozzo P. Chromatic X-Ray imaging with a fine pitch CdTe sensor coupled to a large area photon counting pixel ASIC [arXiv:1210.1248 (physics. ins-det)]. J Instrum. 2012;8:C02028.

27. Pacella D, Pizzicaroli G, Gabellieri L, et al. Ultrafast soft x-ray twodimensional plasma imaging system based on gas electron multiplier detector with pixel readout. Rev Sci Instrum. 2001;72(2):1372-1378.

28. Zentai G. Comparison of CMOS and a-Si flat panel imagers for X-ray imaging. 2011 IEEE International Conference on Imaging Systems and Techniques (IST); May 17-18, 2011; Penang, Malaysia; 2011:194-200.

29. Llopart X, Campbell M, Dinapoli R, San Segundo D, Pernigotti E. Medipix2: a 64-k pixel readout chip with 55- $\mu \mathrm{m}$ square elements working in single photon counting mode. IEEE Trans Nucl Sci. 2002;49(5): 2279-2283.

30. Vincenzi A, De Ruvo PL, Delogu P, et al. Energy characterization of Pixirad-1 photon counting detector system, 16th International Workshop on Radiation Imaging Detectors (iWoRID); June 22-26, 2014; Trieste, Italy.

31. Romano A, Pacella D, Claps G, Causa F, Gabellieri L. Characterization of the C-MOS Cd-Te Imager Pixirad for energy discriminated X-ray imaging. 16th International Workshop on Radiation Imaging Detectors (iWoRID); June 22-26, 2014; Trieste, Italy.

32. Johnson TRC. Dual energy CT: general principle. AJR Am J Roentgenol. 2012;199:53-58.
33. Rebuffel V, Dinten JM. Dual Energy X-ray Imaging: benefits and limits (ECNDT 2006-Th1.3.1). Insight-Non-Destructive Testing Condition Monitoring. 2007;49:589-594.

34. Vlassenbroek A. Dual Layer CT in clinical practice. Med Radiol. 2011:21-34.

35. Langton CM, Njeh CF. The Physical Measurement of Bone. Philadelphia, PA: Institute of Physics; 2004.

36. International Atomic Energy Agency (IAEA). Dual Energy X Ray Absorptiometry for Bone Mineral Density and Body Composition Assessment (IAEA Human Health Series No 15). Vienna, Austria: International Atomic Energy Agency; 2010.

37. Hoheisel M. Review of medical imaging with emphasis on X-ray detectors. Nucl Instrum Methods Phys Res A. 2006;563:215-224.

38. Levin JM, Isaac PK, Vance V, Larke FJ. Dual-energy contrastenhanced digital subtraction mammography: feasibility. Radiology. 2003;229:261-268.

39. Fornaro J, Leschka S, Hibbeln D, et al. Dual and multi-energy CT: approach to functional imaging. Insights Imaging. 2011;2:149-159.

40. Anderson NG. Spectroscopic (multi energy) CT distinguishes iodine and barium contrast material in mice. Eur Radiol. 2010;20:2126-2134.

41. Pacella D, Bellazzini R, Brez A, Pizzicaroli G, Finkenthal M. X-VUV spectroscopic imaging with a micropattern gas detector. Nucl Instrum Methods Phys Res A. 2003;508(3):414-424.

42. Pacella D, Bellazzini R, Brez A, Pizzicaroli G. Fast 2-D soft X-ray imaging device based on micro pattern gas detector. Nucl Phys B. 2003;125:332-336.

43. Pacella D, Bellazzini R, Finkenthal M. Energy resolved two-dimensional soft x-ray radiography with a micropattern gas detector. Rev Sci Instrum. 2006;77(4):043702.

44. Wang X, Meier D, Mikkelsen S, et al. Micro CT with energy-resolved photon counting detectors. Phys Med Biol. 2011;56(9):2791-2816.

45. Butler APH, Andersonb NG, Tipples R, et al. Butler, bio-medical X-ray imaging with spectroscopic pixel detectors. Nucl Instrum Methods Phys Res A. 2008;591:141-146.

46. Tlustos L. Spectroscopic X-ray imaging with photon counting pixel detectors. Nucl Instrum Methods Phys Res A. 2010;623:823-828.

47. Taguchi K, Iwanczyk JS. Vision 20/20: single photon counting x-ray detectors in medical imaging. Med Phys. 2013;40(10):100901.

48. Pacella D, Dabagov S, Murtas F, et al. Polycapillary optics for soft X-ray imaging and tomography. Nuovo Cimento della Societa Italiana di Fisica C. 2011;34(4):513-520.

49. Zhang J. X-ray laser 2004 (Institute of Physics Conference Series Number 186). Proceedings of the 9th International Conference on X-ray Lasers, Beijing, China, May 24-28, 2004

50. PiXirad. Image sensor: principle of operation of pixirad. Available from: http://www.pixirad.com/?page_id=2. Accessed December 22, 2014.

51. PiXirad. Digital image of a rat's thoracic cavity. Available from: http:// www.pixirad.com/?page_id=396. Accessed December 22, 2014.
Reports in Medical Imaging

\section{Publish your work in this journal}

Reports in Medical Imaging is an international, peer-reviewed, open access journal publishing original research, reports, reviews and commentaries on all areas of medical imaging. The manuscript management system is completely online and includes a very quick and fair peer-review system, which is all easy to use.

\section{Dovepress}

Visit http://www.dovepress.com/testimonials.php to read real quotes from published authors. 